\title{
Capsule Commentary on Bauer et al., Health Literacy and Antidepressant Adherence Among Adults with Diabetes: Implications for Future Research
}

\author{
Deena J. Chisolm, PhD \\ J Gen Intern Med 28(9): 1223 \\ DOI: $10.1007 / \mathrm{s} 11606-013-2437-\mathrm{x}$ \\ (c) Society of General Internal Medicine 2013
}

The Ohio State University College of Medicine, Columbus, OH, USA.

$\mathrm{D}$ iabetes affects more than 25.8 million Americans, with 1.9 new adult cases diagnosed in 2010. ${ }^{1}$ While heart disease, stroke, amputation, and blindness are the diabetic outcomes that get the most attention, depression is also common in this population and significantly reduces quality of life. ${ }^{1}$ This paper examines the relationship between health literacy and depression medication adherence in a population already heavily burdened with disease management responsibility. ${ }^{2}$ The authors found that in a population of insured adults with type 2 diabetes, health literacy limitations are associated with multiple measures of antidepressant non-adherence. ${ }^{3}$

The study leaves two important questions. The first is whether the relationship found between antidepressant adherence and health literacy is specific to antidepressants, or if it is part of a larger relationship between health literacy and medication management in patients with diabetes. Previous research on the relationship between health literacy and diabetes medication adherence has generated weak and mixed results, ${ }^{3}$ supporting the unstated hypothesis that the relationship with antidepressants may be stronger than, or at least independent of, that with diabetes-specific medications. A differential relationship between health literacy and adherence to medical regimens supports a combined approach of "universal" health literacy precautions for disease management in general, and depressionspecific communication interventions such as team-based care with care management support.

Secondly, the nesting of this study within a population of diabetic adults raises the question of whether the identified relationship between health literacy and antidepressant adherence is unique to the diabetes population or whether a similar relationship would be seen in depressed patients with other chronic illnesses. This is important because increased rates of major depression have been found across a number of chronic conditions, including end-stage renal disease, chronic obstructive pulmonary disease (COPD), and congestive heart failure, and these depression rates have been associated with poor health outcomes and increased utilization. ${ }^{4}$ This study shows an association between low literacy and reduced adherence to antidepressant medications in patients with diabetes. What remains to be proven is whether addressing health literacy, through proven approaches that reduce patient burden and increase patient competency, can improve adherence, and more importantly, improve outcomes such as health status and costs for chronic disease patients more broadly. Such evidence would provide strong support for greater investment in health literacy programs.

Conflicts of interest: The author declares no conflicts with any of the material in this article.

Corresponding Author: Deena J. Chisolm, PhD; The Ohio State University College of Medicine, Columbus, $\mathrm{OH}$ 43205, USA (e-mail: Deena.Chisolm@NationwideChildrens.org).

\section{REFERENCES}

1. Ali S, Stone M, Skinner T, Robertson N, Davies M, Khunti K. The association between depression and health-related quality of life in people with type 2 diabetes: a systematic review. Diabetes/Metabolism Research and Reviews. 2010;26(2):75-89.

2. Bauer AM, Schillinger D, Parker MM, Katon W, Adler N, Moffet HH, Karter AJ. Health literacy and antidepressant medication adherence among adults with diabetes: The Diabetes Study of Northern California (DISTANCE). J Gen Intern Med. 2013. doi:10.1007/s11606-013-2402-8.

3. Loke Y, Hinz I, Wang X, Salter C. Systematic review of consistency between adherence to cardiavascular or diabetes medication and health literacy in older adults. Annals of Pharamacotherapy. 2012;46(6):863-72.

4. Egede L. Major depression in individuals with chronic medical disorders: Prevalence, correlates and association with health resource utilization, lost productivity and functional disability. General Hospital Psychiatry. 2007;29(5):409-16. 